\title{
Spatial beam nonlinear control with multimode GRIN fiber amplifiers
}

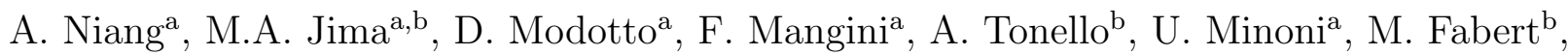 \\ M. Zitellic ${ }^{\mathrm{c}}$ V. Couderc ${ }^{\mathrm{b}}$, and S. Wabnitz ${ }^{\mathrm{c}}$ \\ ${ }^{a}$ DII, University of Brescia, via Branze 38, 25123, Brescia, Italy

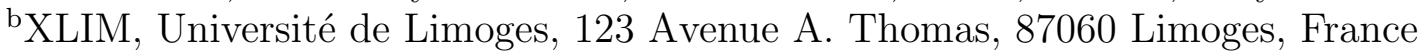 \\ ${ }^{\mathrm{c}}$ DIET, Sapienza University of Rome, Via Eudossiana 18, 00184 Rome, Italy
}

\begin{abstract}
We analyzed the nonlinear dynamics of pulsed beam self-cleaning in nonlinear tapered Ytterbium doped and Erbium-Ytterbium codoped graded-index multimode optical fibers, with quasi-uniform doping distribution in the core cross-section. By increasing the net gain when operating in active configuration we observed that the output spatial intensity distribution changed from a speckled into a high-quality and bell-shaped beam. By launching pulses in the normal dispersion regime of the taper, from the wider into the smaller core diameter, we generated a supercontinuum emission between $520 \mathrm{~nm}$ and $2600 \mathrm{~nm}$. When the laser pulses were launched into the small core side of the tapered fiber or in the Erbium-Ytterbium fiber, self-cleaning was obtained without any self-phase modulation-induced spectral broadening or frequency conversion.
\end{abstract}

Keywords: Fiber nonlinear optics, optical fiber amplifiers, Kerr effect, supercontinuum generation

\section{INTRODUCTION}

Currently, there is a strong fundamental and applicative interest in the use of nonlinear multimode optical fibers (MMFs) for the controlled manipulation of the spectral, spatial, and temporal degrees of freedom of laser beams. ${ }^{1}$ Among MMFs, graded-index (GRIN) fibers exhibit a self-imaging effect, leading to a periodic refractive index modulation induced by the Kerr nonlinearity. ${ }^{2}$ Moreover, GRIN fibers have relatively low modal dispersion, which permits long interaction lengths between different fiber modes. Multimode optical solitons, ${ }^{3}$ geometrical parametric instabilities (GPIs), ${ }^{4}$ Kerr beam self-cleaning (KBSC), ${ }^{5}$ ultra-wide supercontinuum generation $(\mathrm{SCG}),{ }^{6}$ and intermodal four-wave mixing $(\mathrm{IFWM})^{7}$ can be cited among the major recent advances in multimode nonlinear fiber optics.

KBSC is a nonlinear process involving the self-organization of coupled fiber modes. In its first manifestation, KBSC transforms the output random speckled beam at low powers, into a bell-shaped beam close to the fundamental transverse mode at a high powers. ${ }^{5}$ Subsequent experiments have shown that the output transverse spatial distribution at high powers may be controlled, by varying the fiber coupling conditions of the input laser. $^{8}$ Nonlinear pulse propagation and beam reshaping in standard or tapered GRIN MMFs ${ }^{9-11}$ have been investigated in both the normal and in the anomalous dispersion regime, ${ }^{12,13}$ using pulses with duration ranging from sub-nanoseconds to femtoseconds. ${ }^{14-16}$

Active MMFs are key components for a future generation of fiber lasers, which may enable to scale-up by orders of magnitude the peak power of mode-locked laser sources. ${ }^{17}$ Currently, the power delivered by fiber lasers is limited by nonlinear effects such as Raman and Brillouin scattering, as well as by transverse mode instabilities (TMIs). The difficulty arises in simultaneously suppressing both stimulated Raman scattering (SRS) and TMI in a fiber laser system. ${ }^{18}$ Tao et al. have demonstrated the possibility to suppress either SRS or TMI, by shifting the pump wavelength, ${ }^{19}$ or by optimizing the longitudinal pump power distribution along the active fiber. ${ }^{20}$

TMI is a main limiting factor for large mode area (LMA) CW fiber lasers, which leads to sudden power fluctuations, and to a reduction of the output beam quality at the amplifier output. ${ }^{21}$ To mitigate the effects

Further author information: alioune.niang@unibs.it 
of TMI, a possible solution is to use tapered Ytterbium-doped (T-YD) fibers, where the core diameter of the fiber varies along its axis. When the signal propagates from the small to the large core side of the taper, T-YD fibers permit to filter out high-order modes (HOMs), hence maintaining a good beam quality along the fiber. In addition, the progressively increasing mode area permits to suppress nonlinear effects, such as frequency conversion processes leading to a degradation of the beam quality.

The aim of this work is to show that it is possible to exploit the nonlinear effect of beam self-cleaning, in order to overcome the beam quality degradation in active MMFs. Beam self-cleaning was demonstrated in quasi-step index core refractive index YD MMFs, leading to measured $M^{2}$ parameters around $2 .{ }^{22}$ Here, we demonstrate Kerr beam self-cleaning in two different types of active MMFs with a parabolic (GRIN) core refractive index profile, and quasi-uniform doping distribution. First, we used a T-YD GRIN-MMF with a core diameter varying exponentially along the longitudinal direction. ${ }^{10,11}$ By propagating pulses at $1064 \mathrm{~nm}$ from the largest end of the T-YD MMF, amplification permits a combination of beam self-cleaning and SCG, spanning from the visible to the mid-infrared. In order to use T-YD MMFs in a fiber laser, frequency conversion phenomena should be avoided: therefore, the amplified laser signal must necessarily propagate from the smaller to the larger core diameter. For this reason, we launched a beam into the smaller end of the Y-TD fiber. This configuration leads to amplification preserving a high beam quality, without any frequency conversion. The last type of active MMF that we considered consists of an Erbium-Ytterbium co-doped (EYD) GRIN fiber. With this fiber amplifier, we also could obtain beam self-cleaning with a record good beam quality of $M^{2}=1.6$.

\section{SELF-CLEANING IN GRIN T-YD MMFS}

The main mechanism behind spatiotemporal nonlinear processes in a GRIN MMFs is the periodic self-imaging effect, and the associated periodic refractive index modulation induced by the Kerr nonlinearity. Since the selfimaging period is directly proportional to the core diameter, by tapering a GRIN MMF one has an additional degree of freedom in controlling the spectral and spatial properties of a propagating beam. Indeed, injecting a beam into the large core side of the taper accelerates both self-imaging and nonlinear interactions, as the core diameter decreases. This results in broadband, multimode SCG, as reported by Eftekhar et al. for the case of a multimode passive (undoped) GRIN tapered fiber. ${ }^{9}$ On the other hand, when the beam propagates from the small to the large core side, the nonlinear coefficient decreases as the core diameter increases, and decelerated self-imaging occurs. In an active tapered MMF, the presence of a decelerating nonlinearity permits to amplify the signal without any accompanying frequency conversion. On the other hand, the capability of still nonlinearly cleaning the beam, even in the presence of a decelerating nonlinearity, would provide a considerable progress for multimode fiber amplifier and laser applications.

In our experiments, we used a specially designed Ytterbium-doped multimode fiber taper with a parabolic refractive index profile, a core diameter that is exponentially varying along its length, and a quasi-uniform doping distribution in the core cross-section. Fig. 1 shows the characteristics of our tapers. The tapers are used in different series of experiments, in order to achieve spatial beam self-cleaning in active (i.e., with gain from the pump laser diode (LD)) configuration. A pulsed signal beam at $1064 \mathrm{~nm}$, thus propagating in the normal dispersion regime of the fiber taper, was launched from the wider (smaller) core diameter, so that it experiences accelerating (decelerating) nonlinearity along with its propagation.

\subsection{Large-to-Small (L-S) core configuration}

Our first series of experiments involved the use of sub-nanosecond signal pulses, launched from the large core side of the taper and emerging from the small core side. The tapered Yb-doped MMF had a $9.5 \mathrm{~m}$ length, and a core diameter varying from 122 to $37 \mu \mathrm{m}$ (the square cladding had side varying from $350 \mu \mathrm{m}$ to $90 \mu \mathrm{m}$ ). For this configuration, the schematic of the experimental setup is represented in Ref. ${ }^{10}$ For the signal, we used a Nd:YAG microchip laser at $1064 \mathrm{~nm}$, with Gaussian spatial beam shape, generating 500 ps pulses at the repetition rate of $500 \mathrm{~Hz}$. Moreover, a multimode CW pump LD with up to $20 \mathrm{~W}$ power at $940 \mathrm{~nm}$ was cladding coupled to the active taper in a co-propagation geometry. The output beam was imaged with a micro-lens on a CCD camera and an optical spectrum analyzer (OSA), to monitor its spatial and spectral distributions, respectively.

We started our experiments by switching off the pump LD, and by setting the signal input peak power at $19.6 \mathrm{~kW}$. In this situation the signal experienced significant linear loss: as a result, the output beam was not 
(a)
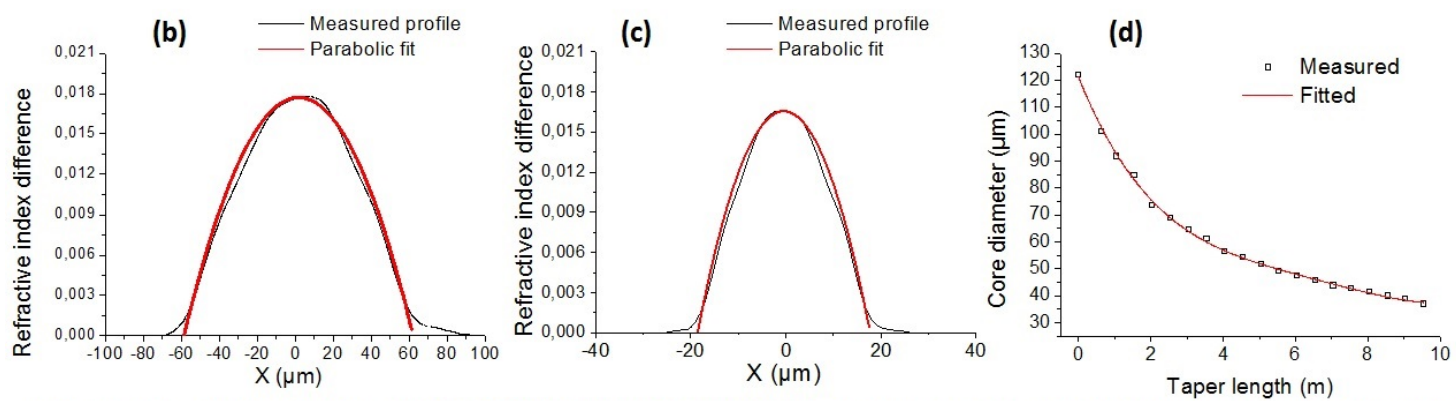

Figure 1. Characteristics of our taper: (a) Tapered Ytterbium doped MMF, (b) and (c) profile of the refractive index difference between core and cladding for either the large or the small core diameter, respectively, and (d) core diameter of tapered fiber vs. taper length.

cleaned up, see Fig. 2(a). Next, we turned on the pump LD. By increasing the gain provided by the pump, we observed a progressive output beam shaping into flat-top cleaned spatial transverse profile. The corresponding spatial intensity distribution is presented in Fig. 2(b-h). The net gain threshold for the beam cleaning effect to appear is $G=0.2$ ( $G$ is the ratio of output to input coupled signal average power at $1064 \mathrm{~nm}$ and we used the same definition in our experiments); beam self-cleaning preserved up to $G=1.34$ (maximum net gain value). Owing to the non-monotonic variation of the average beam power along the taper, the net gain $\mathrm{G}$ remains low. The signal is only amplified over the first few meters of the taper, and subsequently it is reabsorbed upon its propagation (see Fig. 3(b)). Note that a spectral narrow bandpass filter at $1064 \mathrm{~nm}$ was used in front of the camera, in order to block residual radiation from the pump.
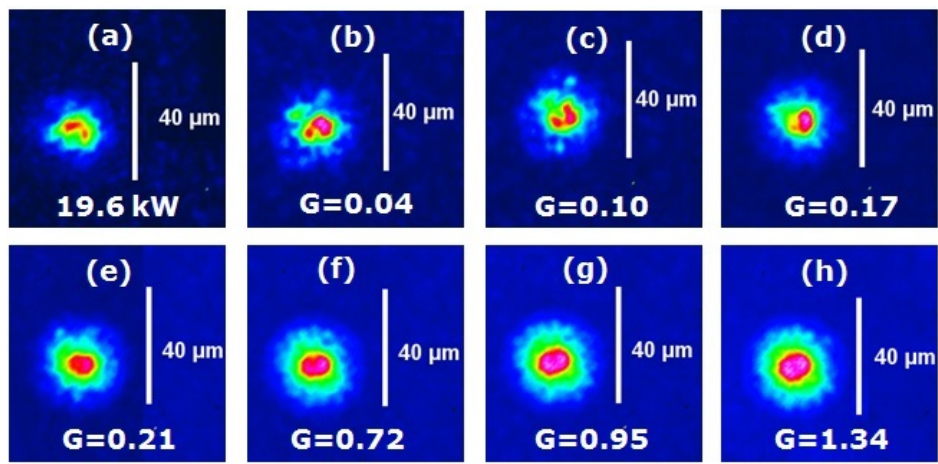

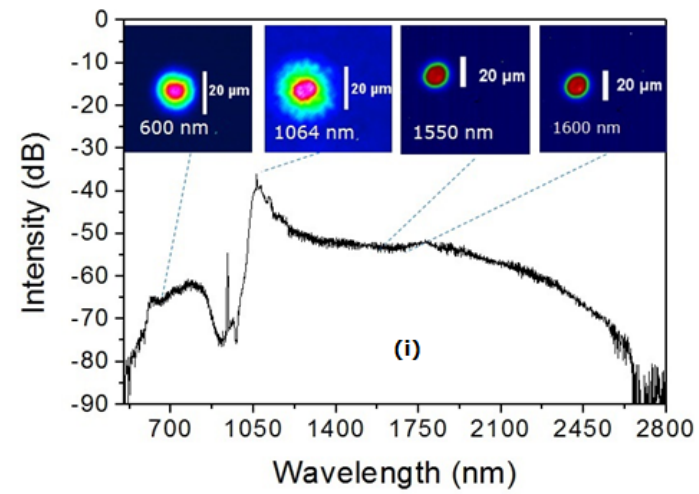

Figure 2. (L-S) configuration: $9.5 \mathrm{~m}$ tapered fiber. Near-field output beam profile (a) without pump LD and (b-h) with different gain $\mathrm{G}$ values. (i) Output spectrum for maximum gain value $\mathrm{G}=1.34$. Inset of (i): near-field output beam profile from bandpass filters with different center wavelengths.

Next, we studied the spectral evolution of the output pulses from the amplifier, as a function of its gain G. At low gain values, the signal is amplified without showing significant spectral broadening. With increasing gain values, a stimulated Raman Stokes line appears together with GPI-generated anti-Stokes sidebands, ultimately leading to an ultra-broadband spectrum. The output spectrum obtained for the maximum gain value is presented in Fig. 2(i). As it can be seen, SCG from visible to mid-infrared $(520-2600 \mathrm{~nm})$ is observed. Our results are in qualitative agreement with those reported for lossless (undoped) GRIN MMF tapers. ${ }^{9}$ The SCG process results from the interplay between Raman scattering, soliton-self-frequency shift, and spatiotemporal instabilities (or GPI). In order to verify if the beam self cleaning exists across each wavelength of the supercontinum, we used different bandpass filters and appropriate cameras in order to visualize the beam profile. As shown in the insets 
(a)

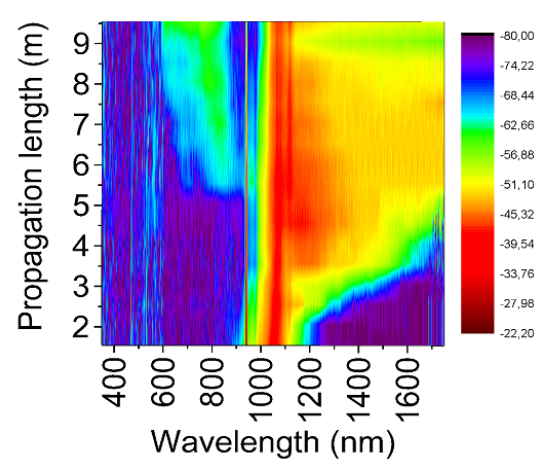

(b)

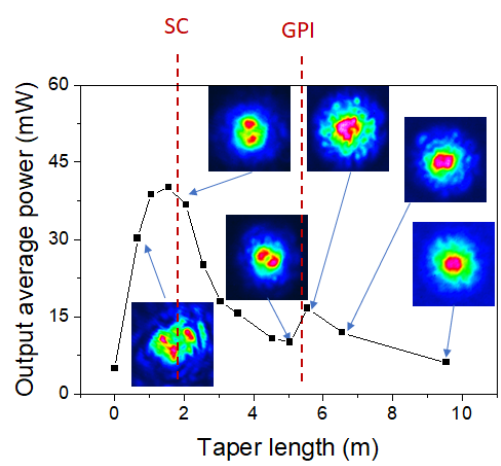

Figure 3. (L-S) configuration: cut-back measurements. (a) Output spectrum vs. propagation length. (b) Output average power at $1064 \mathrm{~nm}$ vs. taper length, showing the evolution of beam self-cleaning in the taper. Insets: output near-field spatial distributions along the taper.

of Fig. 2(i), the effective beam self-cleaning appears at different SC wavelengths.

As a last step, we studied the evolution of KBSC and SCG along the active taper, by means of cutback measurements. We fixed the pump LD at the value leading to maximum gain $(G=1.34)$. We equally cut off the taper from the small face end, at different intermediate positions. Fig. 3(a) summarizes the spectral evolution as a function of taper length. The spectrum broadens after two meters, leading to progressive SCG broadening towards the infrared. Whereas, the first anti-Stokes GPI sideband appears around $6 \mathrm{~m}$. Accelerating self-imaging in the taper leads to a progressive blue-shift of GPI. ${ }^{9,10}$ In Fig. 3(b) we illustrate the evolution of the average output power at $1064 \mathrm{~nm}$, as a function of taper length. As it can be seen, the average beam power varies non-monotonically along the taper, due to modal transitions of self-cleaned beams along the taper. The output near-field spatial beam distributions along the taper are presented in the insets. The beam shape is speckled at the beginning of signal propagation, that is over the first meter, and it is cleaned up to a flat-top profile after $6 \mathrm{~m}$. Between these two shapes, the self-cleaning on $L P_{11}$ mode appears as a transient mode-cleaning effect. On the other hand, the length of the taper, which leads to maximum amplification of the input signal (optimal length), is around $1.55 \mathrm{~m}$. Thus, nonlinear effects are enhanced after $2 \mathrm{~m}$ of taper, which effectively turns on Raman gain, and its associated spectral broadening. Note that the vertical dashed red lines in Fig. 3(b) correspond to the positions of GPI and SCG threshold in the taper.

\subsection{Small-to-Large (S-L) core configuration}

In the second and last series of taper experiments, the signal at $1064 \mathrm{~nm}$ was launched into the smallest end of the taper, a configuration which leads to decelerating nonlinearity. Here, the input signal is identical to that used in the previous study, but with a peak power up to $88 \mathrm{~kW}$. Whereas a $10 \mathrm{~W}$ output power CW multimode $\mathrm{LD}$ at $940 \mathrm{~nm}$ was cladding-coupled in a counter-propagation configuration. In this study, we used a $5 \mathrm{~m}$ of tapered fiber: the T-YD MMF had a core diameter of 47 and $96 \mu \mathrm{m}$ for its small and large ends, respectively. The corresponding experimental setup is described and presented in Ref. ${ }^{11}$

At first, we characterised our tapered amplifier by studying the amplified spontaneous emission (ASE) noise, and the amplification of the $1064 \mathrm{~nm}$ signal as a function of the pump LD power. Fig. 4(a) shows the ASE noise power vs. LD pump power, at it was measured after a bandpass filter centered at $1064 \mathrm{~nm}$ (with $10 \mathrm{~nm}$ bandwidth) at both ends of the taper. The highest ASE noise power was obtained from the large core diameter end-face. Fig. 4(b) reports the signal Gain for different input signal peak power levels. As we can see, the amplification is strongly saturated, being limited to $\mathrm{G} \simeq 1$ as the signal peak power increases up to $10 \mathrm{~kW}$.

Next, we studied spatial beam self-cleaning in the decelerating nonlinearity T-Yb amplifier. Signal input peak power was fixed at $10 \mathrm{~kW}$, that is below the self-cleaning threshold in the passive configuration. By placing a bandpass filter (FWHM $3 \mathrm{~nm}$ ) before the camera, we visualized the evolution of the output signal beam at $1064 \mathrm{~nm}$, as a function of the pump LD power. The obtained near-field spatial beam patterns are presented 
(a)

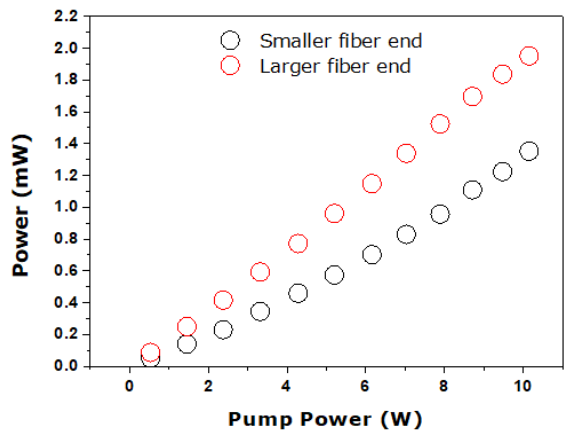

(b)

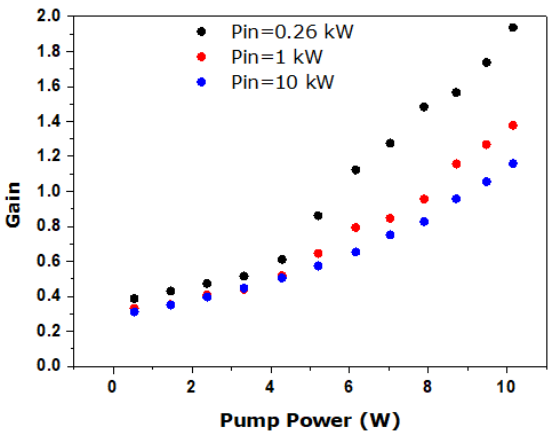

Figure 4. (S-L) configuration: (a) amplification of ASE noise vs. pump LD power. (b) net signal gain G vs. pump LD power, for different signal peak powers.

(a)

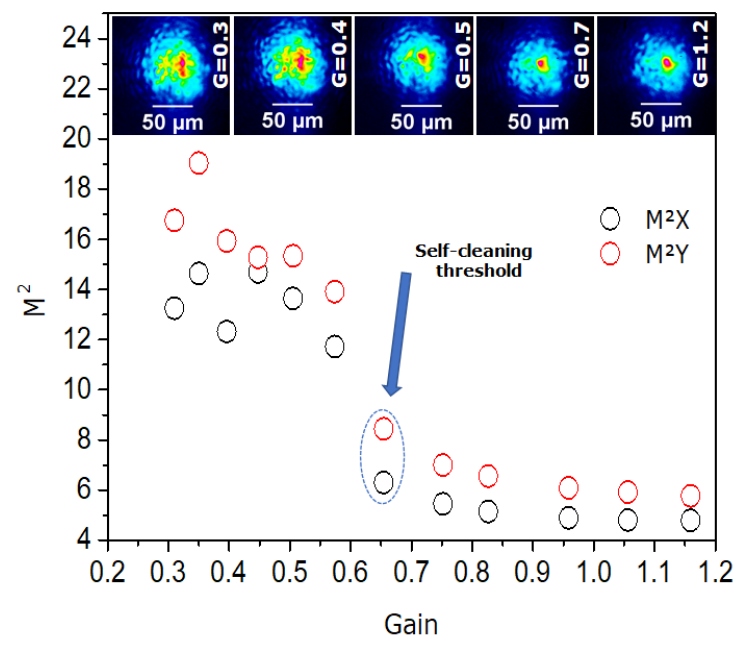

(b)

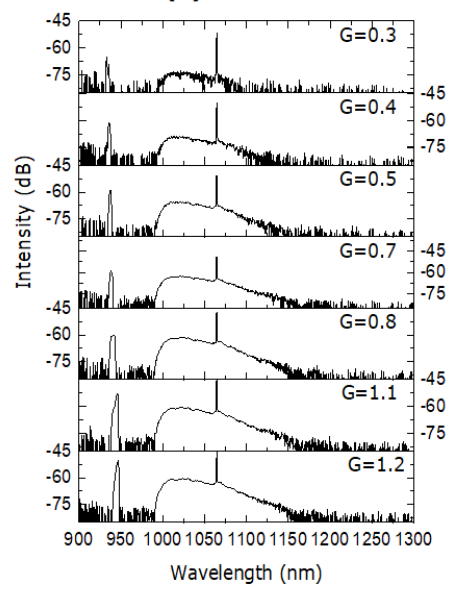

Figure 5. (S-L) configuration: (a) Beam quality $M^{2}$ parameter vs. Gain; insets: near-field spatial distributions for different gain values. (b) Spectral distributions.

in the insets of Fig. 5(a). As it can be seen, the output beam is highly speckled. Increasing the gain up to $\mathrm{G}=0.7$ leads to obtaining self-cleaning. The output beam remains cleaned even if the gain is further increased. In addition, we measured the near-field and far-field output beam diameters at $1 / e^{2}$ of the maximum intensity, in order to quantitatively verify the beam quality at the taper output. We calculated the gain dependence of the beam quality factor $M^{2}$ of the output beam (along its two orthogonal transverse directions). As it can be seen in Fig. 5(a), the $M^{2}$ beam quality parameter drops from 19 (for 0.3 ) down to 5 for the maximum gain of $\mathrm{G}=1.2$. Note that the $M^{2}$ value for spatial beam self-cleaning at the same wavelength in a standard lossless GRIN fiber is nearly equal to four. ${ }^{5}$

The output spectra of pulses that undergo spatial beam self-cleaning are illustrated in Fig. 5(b). As it can be seen, in this case self-cleaning is not accompanied by any significant spectral broadening, contrary to the previous case where the signal was injected from the large core side of the taper (accelerating nonlinearity). In addition to the spectral line pertaining to the back-reflected $940 \mathrm{~nm}$ pump LD, Fig. 5(b) only shows the presence of a wideband ASE background surrounding the relatively narrow spectral line of the signal. 
(a)

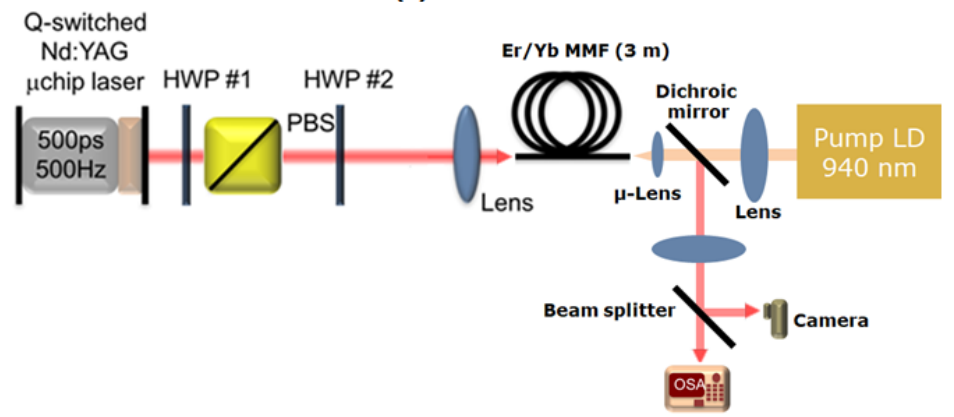

(b)

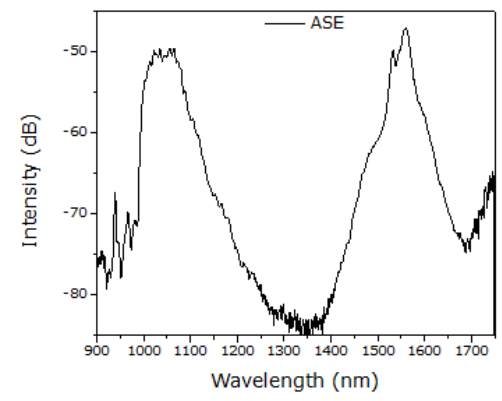

Figure 6. (a) Schematic of the experimental setup for self-cleaning in an Er-Yb amplifier. (b) ASE spectrum of Er-Yb co-doped fiber.

\section{BEAM SELF-CLEANING IN A GRIN EYD MMF}

In this Section, we present the experimental study of beam self-cleaning in a specially designed GRIN EYD MMF amplifier, with quasi-uniform doping distribution in the core cross-section. The fiber was doped with a mass concentration of 1.4 and $0.6 \%$ for Er and $\mathrm{Yb}$, respectively.

For studying the nonlinear dynamics of beam propagation in a GRIN EYD amplifier, we used the same Gaussian spatial beam shape at $1064 \mathrm{~nm}$, as described in Section 2. The schematic of the experimental setup is presented in Fig. 6(a). A polarizing beam splitter and two half-wave plates were combined to adjust the input peak power and polarization state of the signal. The signal was focused with a beam diameter of $14 \mu \mathrm{m}$ at full width of half maximum intensity (FWHMI) into a $3 \mathrm{~m}$ long Er-Yb MMF, with a core diameter of $65 \mu \mathrm{m}$ and square cladding with $200 \mu \mathrm{m}$ side. A pump LD up to $20 \mathrm{~W}$ of power was injected into the opposite side of the active MMF. A dichroic mirror was used to couple the pump LD, and to extract the output beam, which was visualized with a CCD camera and an OSA, to monitor spatial and spectral distributions, respectively.

The ASE spectrum of Er-Yb co-doped fiber for the maximum pump LD power is presented in Fig. 6(b). The co-doped fiber has gain around both $1064 \mathrm{~nm}$ and $1550 \mathrm{~nm}$. Both gain bands extend over approximately $180 \mathrm{~nm}$ (at $-20 \mathrm{~dB}$ ), however, the intensity of gain band around $1550 \mathrm{~nm}$ is $2.5 \mathrm{~dB}$ more than the one around $1064 \mathrm{~nm}$. In addition, the spectral trace shows back-reflected light the pump LD at $940 \mathrm{~nm}$.

For the beam self-cleaning experiments, we started by measuring the self-cleaning threshold in the passive configuration, i.e., with the pump LD switched off, as done for the tapered YD amplifiers. The measured beam self-cleaning input signal power threshold was around $5 \mathrm{~kW}$. Next, we fixed the input signal peak power at 1.64 $\mathrm{kW}$, and characterized the output spatial intensity distributions as a function of the pump LD power. As it can be seen in Fig. 7(a), the amplified beam maintained a good, nearly singlemode, quality when the amplifier gain was increased up to $6 \mathrm{~dB}(\mathrm{G}=4)$. For low net gain $\mathrm{G}$ values, the spatial output beam pattern was speckled. Thus increasing the pump power (gain) leads to a self-organization and cleanup of the output transverse intensity distribution. The beam quality $\left(M^{2}\right)$ of the output beam was also measured as a function of the net gain value, see Fig. 7(b). The $M^{2}$ factor is reduced down to approximately 1.7 when self-cleaning appears, and it drops to 1.6 for the maximum gain value.

Finally, we measured the evolution of the spectral distribution as a function of the amplifier gain, that accompanies the beam self-cleaning effect. We obtained that the output spectrum does not experience significant nonlinear spectral broadening, even for gain values leading to the beam self-cleaning. The output spectrum with maximum gain $(\mathrm{G}=4)$ is shown in Fig. $7(\mathrm{c})$ : the ASE noise with the same pump LD power is given for comparison. As it can be seen, beam self-cleaning is not accompanied by frequency conversion processes.

\section{CONCLUSIONS}

To conclude, we studied nonlinear spatial beam reshaping in both tapered T-YD and quasi-uniform EYD GRIN MMFs amplifiers. These special amplifiers where specially designed and fabricated to have a parabolic gradedindex profile, and a nearly uniform doping distribution across the core. In both multimode amplifiers, we 

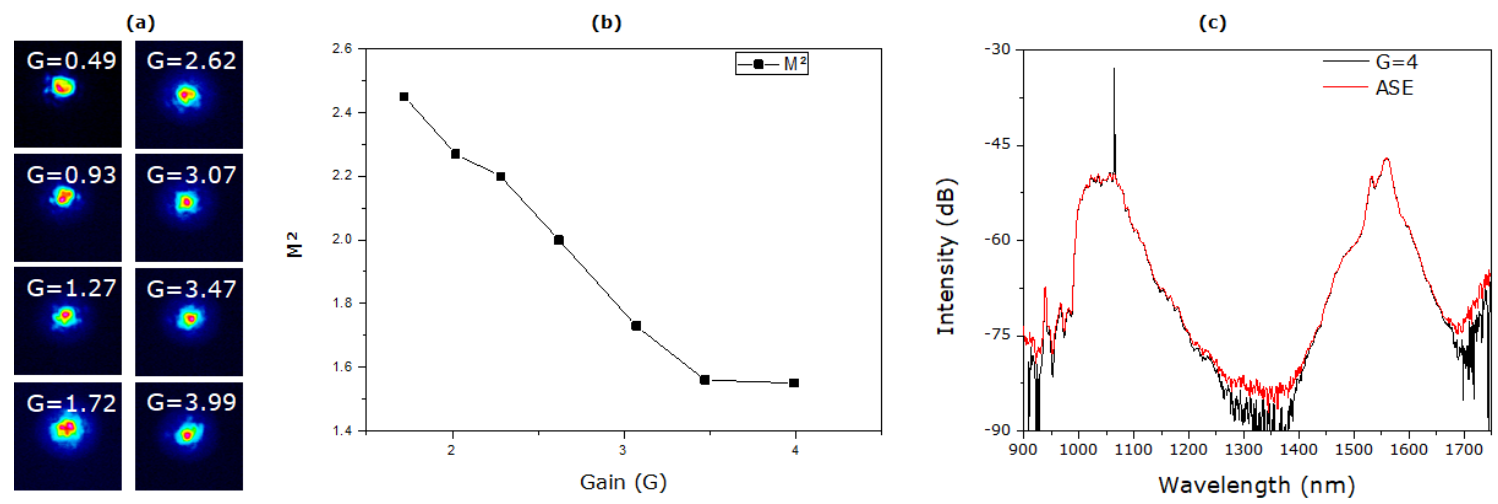

Figure 7. GRIN EYD MMF: a) Near field spatial intensity distribution. b) Beam quality $M^{2}$ parameter vs. gain. (c) Spectrum evolution for $\mathrm{G}=4$ with the ASE signal.

demonstrated that beam self-cleaning can be achieved by appropriately increasing the signal or the pump power above a given threshold. In the T-YD GRIN-MMF with accelerating nonlinearity, gain permits to combine self-cleaning with a wideband SCG, extending from 520 to $2600 \mathrm{~nm}$. On the other hand, when the signal were launched into the EYD fiber or into the small core side of the YD taper, one obtains beam self-cleaning with accompanying high beam quality recovery. Specifically, beam quality factors of $M^{2}=5$ and $M^{2}=1.6$ were obtained with either tapered or EYD fibers, respectively. Our experimental results confirm that the presence of the gain (pump) in the taper or in EYD GRIN MMFs amplifiers allows for efficient nonlinear self-organization of the transverse spatial beam into a high quality transverse intensity profile. The presence of beam self-cleaning in multimode GRIN fiber amplifiers could pave the way for developing a new class of high-beam-quality, and high-power fiber lasers and amplifiers.

\section{ACKNOWLEDGMENTS}

We thank O.N. Egorova, A.E. Levchenko, and S.L. Semjonov from the Fiber Optics Research Center of the Russian Academy of Sciences (RAS), and D.S. Lipatov from the Devyatykh Institute of Chemistry of HighPurity Substances of RAS, for providing the tapered YD and EYD GRIN MMF.

We acknowledge financial support from the European Research Council (grant No. 740355) and the Italian Ministry of Education, University and Research (MIUR) under PRIN 2015 NEMO project (grant No. 2015KEZNYM); Xcore research foundation; French program 364 "Investissement d'Avenir" ((ANR-15 IDEX0003), Project No. ISITE-BFC-299365); CILAS company (Ariangroup) and Russian Science Foundation (RSF) (grant no. 17-13-01343).

\section{REFERENCES}

[1] Krupa, K., Tonello, A., Barthélémy, A., Mansuryan, T., Couderc, V., Millot, G., Grelu, P., Modotto, D., Babin, S. A., and Wabnitz, S., "Multimode nonlinear fiber optics, a spatiotemporal avenue," $A P L$ Photonics 4(11), 110901 (2019).

[2] Hansson, T., Tonello, A., Mansuryan, T., Mangini, F., Zitelli, M., Ferraro, M., Niang, A., Crescenzi, R., Wabnitz, S., and Couderc, V., "Nonlinear beam self-imaging and self-focusing dynamics in a grin multimode optical fiber: theory and experiments," Opt. Express 28, 24005-24021 (Aug 2020).

[3] Renninger, W. H. and Wise, F. W., "Optical solitons in graded-index multimode fibres," Nat. Commun. 4, 1719 (2013).

[4] Krupa, K., Nithyanandan, K., Andral, U., Tchofo-Dinda, P., and Grelu, P., "Real-time observation of internal motion within ultrafast dissipative optical soliton molecules," Phys. Rev. Lett. 118, 243901 (Jun 2017).

[5] Krupa, K., Tonello, A., Shalaby, B. M., Fabert, M., Barthélémy, A., Millot, G., Wabnitz, S., and Couderc, V., "Spatial beam self-cleaning in multimode fibres," Nat. Photonics 11, 237-241 (2017). 
[6] Krupa, K., Louot, C., Couderc, V., Fabert, M., Guenard, R., Shalaby, B. M., Tonello, A., Pagnoux, D., Leproux, P., Bendahmane, A., Dupiol, R., Millot, G., and Wabnitz, S., "Spatiotemporal characterization of supercontinuum extending from the visible to the mid-infrared in a multimode graded-index optical fiber," Opt. Lett. 41, 5785-5788 (Dec 2016).

[7] Bendahmane, A., Krupa, K., Tonello, A., Modotto, D., Sylvestre, T., Couderc, V., Wabnitz, S., and Millot, G., "Seeded intermodal four-wave mixing in a highly multimode fiber," J. Opt. Soc. Am. B 35, 295-301 (Feb 2018).

[8] Deliancourt, E., Fabert, M., Tonello, A., Krupa, K., Desfarges-Berthelemot, A., Kermene, V., Millot, G., Barthélémy, A., Wabnitz, S., and Couderc, V., "Kerr beam self-cleaning on the lp11 mode in graded-index multimode fibers," OSA Continuum 2, 1089-1096 (Apr 2019).

[9] Eftekhar, M. A., Sanjabi-Eznaveh, Z., Lopez-Aviles, H. E., Benis, S., Antonio-Lopez, J. E., Kolesik, M., Wise, F., Amezcua-Correa, R., and Christodoulides, D. N., "Accelerated nonlinear interactions in gradedindex multimode fibers," Nature Communications 10(1), 1638 (2019).

[10] Niang, A., Mansuryan, T., Krupa, K., Tonello, A., Fabert, M., Leproux, P., Modotto, D., Egorova, O. N., Levchenko, A. E., Lipatov, D. S., Semjonov, S. L., Millot, G., Couderc, V., and Wabnitz, S., "Spatial beam self-cleaning and supercontinuum generation with yb-doped multimode graded-index fiber taper based on accelerating self-imaging and dissipative landscape," Opt. Express 27, 24018-24028 (Aug 2019).

[11] Niang, A., Modotto, D., Tonello, A., Mangini, F., Minoni, U., Zitelli, M., Fabert, M., Jima, M. A., Egorova, O. N., Levchenko, A. E., Semjonov, S. L., Lipatov, D. S., Babin, S., Couderc, V., and Wabnitz, S., "Spatial beam self-cleaning in tapered yb-doped grin multimode fiber with decelerating nonlinearity," IEEE Photonics Journal 12(2), 1-8 (2020).

[12] Krupa, K., Castañeda, G. G., Tonello, A., Niang, A., Kharenko, D. S., Fabert, M., Couderc, V., Millot, G., Minoni, U., Modotto, D., and Wabnitz, S., "Nonlinear polarization dynamics of Kerr beam self-cleaning in a graded-index multimode optical fiber," Opt. Lett. 44, 171-174 (Jan 2019).

[13] Leventoux, Y., Parriaux, A., Sidelnikov, O., Granger, G., Jossent, M., Lavoute, L., Gaponov, D., Fabert, M., Tonello, A., Krupa, K., Desfarges-Berthelemot, A., Kermene, V., Millot, G., Février, S., Wabnitz, S., and Couderc, V., "Highly efficient few-mode spatial beam self-cleaning at 1.5\&\#x00b5;m," Opt. Express 28, 14333-14344 (May 2020).

[14] Wright, L. G., Liu, Z., Nolan, D. A., Li, M.-J., Christodoulides, D. N., and Wise, F. W., "Self-organized instability in graded-index multimode fibres," Nat. Photonics 10, 771-776 (November 2016).

[15] Krupa, K., Tonello, A., Barthélémy, A., Couderc, V., Shalaby, B. M., Bendahmane, A., Millot, G., and Wabnitz, S., "Observation of geometric parametric instability induced by the periodic spatial self-imaging of multimode waves," Phys. Rev. Lett. 116, 183901 (May 2016).

[16] Liu, Z., Wright, L. G., Christodoulides, D. N., and Wise, F. W., "Kerr self-cleaning of femtosecond-pulsed beams in graded-index multimode fiber," Opt. Lett. 41, 3675-3678 (Aug 2016).

[17] Wright, L. G., Christodoulides, D. N., and Wise, F. W., "Spatiotemporal mode-locking in multimode fiber lasers," Science 358(6359), 94-97 (2017).

[18] Hejaz, K., Shayganmanesh, M., Rezaei-Nasirabad, R., Roohforouz, A., Azizi, S., Abedinajafi, A., and Vatani, V., "Modal instability induced by stimulated raman scattering in high-power yb-doped fiber amplifiers," Opt. Lett. 42, 5274-5277 (Dec 2017).

[19] Tao, R., Ma, P., Wang, X., Zhou, P., and Liu, Z., "Mitigating of modal instabilities in linearly-polarized fiber amplifiers by shifting pump wavelength," Journal of Optics 17, 045504 (mar 2015).

[20] Tao, R., Ma, P., Wang, X., Zhou, P., and Liu, Z., "Theoretical study of pump power distribution on modal instabilities in high power fiber amplifiers," Laser Physics Letters 14, 025002 (dec 2016).

[21] Jauregui, C., Limpert, J., and Tünnermann, A., "High-power fibre lasers," Nat. Photonics 7, 861-867 (2013).

[22] Guenard, R., Krupa, K., Dupiol, R., Fabert, M., Bendahmane, A., Kermene, V., Desfarges-Berthelemot, A., Auguste, J. L., Tonello, A., Barthélémy, A., Millot, G., Wabnitz, S., and Couderc, V., "Kerr self-cleaning of pulsed beam in an ytterbium doped multimode fiber," Opt. Express 25, 4783-4792 (Mar 2017). 